Rev. Int. Contam. Ambie. 36 (4) 847-856, 2020

https://doi.org/10.20937/RICA.53614

\title{
GEODISPONIBILIDAD DE ELEMENTOS POTENCIALMENTE TÓXICOS EN SUELOS AGRÍCOLAS QUE REPRESENTAN RIESGO PARA EL AMBIENTE Y LA SALUD DE LA POBLACIÓN DEL NEVADO DE TOLUCA, MÉXICO
}

Geoavailability of potentially toxic elements in agricultural soils that represent a risk to the environment and the health of the population of the Nevado de Toluca, Mexico

\author{
Germán MARTÍNEZ-ALVA ${ }^{1}$, Yaqueline Antonia GHENO-HEREDIA ${ }^{2}$, \\ Patricia VIEYRA-REYES ${ }^{1}$, Ángel Roberto MARTÍNEZ-CAMPOS ${ }^{3}$, \\ Julieta CASTILLO-CADENA ${ }^{4}$, Jerónimo Amado LÓPEZ-ARRIAGA ${ }^{1}$, \\ María de los Ángeles MANZUR-QUIROGA ${ }^{5}$ y Tizbe Teresa ARTEAGA-REYES ${ }^{3 *}$
}

${ }^{1}$ Facultad de Medicina, Universidad Autónoma del Estado de México, Paseo Tollocan esq. Jesús Carranza s/n, 50180 Toluca, Estado de México, México.

${ }^{2}$ Facultad de Ciencias Biológicas y Agropecuarias, Universidad Veracruzana, Camino Peñuela-Amatlán s/n, 94945 Amatlán, Veracruz, México.

${ }^{3}$ Instituto de Ciencias Agropecuarias y Rurales, Universidad Autónoma del Estado de México, El Cerrillo Piedras Blancas, 50090 Toluca, Estado de México, México.

${ }^{4}$ Clínica Multidisciplinaria de Salud, Universidad Autónoma del Estado de México, Jesús Carranza 205, 50130 Toluca, Estado de México, México.

${ }^{5}$ Plantel Cuauhtémoc de la Escuela Preparatoria, Universidad Autónoma del Estado de México, H. Colegio Militar esq. Diego Rivera s/N, 50020 Toluca, Estado de México, México.

*Autor para correspondencia: tizbe@hotmail.com

(Recibido: mayo de 2019; aceptado: febrero de 2020)

Palabras clave: plaguicidas, contaminación, salud humana.

\section{RESUMEN}

En el Nevado de Toluca, México, el uso indiscriminado de plaguicidas para la producción de alimentos (principalmente papa) ha generado impactos negativos en las propiedades físicas y químicas del suelo, lo que representa un riesgo inminente para la salud ambiental y humana. El objetivo del presente estudio fue evaluar la concentración total y la fracción geodisponible de los principales elementos potencialmente tóxicos (As, $\mathrm{Pb}$ y $\mathrm{Cd}$ ) provenientes de los plaguicidas más utilizados en suelos agrícolas del Nevado de Toluca. La determinación de metales pesados ( $\mathrm{Pb}$ y $\mathrm{Cd}$ ) se realizó mediante espectroscopia de emisión atómica por plasma de acoplamiento inductivo y para As, mediante espectroscopia de absorción atómica por generación de hidruros. Los resultados mostraron una concentración total considerable de elementos potencialmente tóxicos en la zona, así como una ostensible geodisponibilidad. Por tanto, hay evidencia de riesgo en el corto plazo por el abuso de plaguicidas en esta importante región del Nevado de Toluca, México.

Key words: pesticides, pollution, human health. 


\begin{abstract}
In the Nevado de Toluca, Mexico the indiscriminate use of pesticides for food production (principally potato) has generated negative impacts on the physical and chemical properties of soil, which represents an imminent risk to environmental and human health. The objective of this study was to evaluate the total concentration and geoavailability fraction of the main potentially toxic elements ( $\mathrm{As}, \mathrm{Pb}$ and $\mathrm{Cd}$ ) from the most used pesticides in agricultural soils of the Nevado de Toluca. The determination of heavy metals $(\mathrm{Pb}$ and $\mathrm{Cd})$ was carried out by atomic emission spectroscopy with inductive coupling and in the case of the As, by atomic absorption spectroscopy with hydride generation. The results revealed a considerable total concentration of potentially toxic elements in the area whose geoavailability is evident. Therefore, there is evidence of global in the short term by the abuse of pesticides in this important region of the Nevado of Toluca, Mexico.
\end{abstract}

\section{INTRODUCCIÓN}

La producción agrícola con plaguicidas (estrategia agrícola productiva intensiva y semiintensiva) que se practica actualmente en el contexto nacional e internacional, representa una estrategia insostenible para las comunidades y un riesgo potencial para la salud del ecosistema y las poblaciones humanas (Haro et al. 2002, Bolognesi 2003, Garry 2004, Waliszewski et al. 2004, Bull et al. 2006, Cavallo et al. 2006, Ismail et al. 2008).

La producción de alimentos que requiere el uso moderado o inmoderado de plaguicidas representa per se una situación de riesgo potencial para las comunidades y el mantenimiento de los recursos naturales, situación que debe ser atendida con prioridad por la comunidad científica y los encargados de la toma de decisiones (Bolognesi 2003, Mansour 2004, Hernández et al. 2006).

El uso de plaguicidas, los cuales se definen como "sustancias o mezclas de sustancias que tienen la finalidad de prevenir, destruir, controlar o repeler plagas, incluidos los vectores de enfermedades humanas y de animales, así como las especies no deseadas que dañan o interfieren con la producción agropecuaria y forestal" (USEPA 2010), es un factor que provoca impactos negativos sobre los recursos naturales (agua, suelo, aire, flora y fauna, entre otros). No obstante, el suelo es el que recibe el mayor impacto negativo cuando se incorporan agentes químicos degradantes como los plaguicidas, principalmente a nivel de sus propiedades bioquímicas (biomasa microbiana edáfica) y químicas ( $\mathrm{pH}$, materia orgánica y capacidad de intercambio catiónico, entre otras), lo que provoca su continuo agotamiento biogeoquímico.

El suelo es considerado un recurso primario que ha permitido sostener la vida como la conocemos.
Es el sustrato básico para las plantas y, por ende, de la producción masiva de alimentos. Capta, retiene y emite agua, y es un filtro ambiental efectivo (Boul 2005). El desarrollo e implementación de estrategias que eviten o reduzcan los procesos y riesgos de degradación, e incrementen el potencial productivo sostenible del suelo para satisfacer las necesidades de los organismos y la sociedad misma (los cuales dependen de dicho recurso) ha sido una de las prioridades de investigación para la comunidad científica. Esto justifica tener una concepción objetiva del concepto "calidad y sostenibilidad del suelo", ya que tanto su comprensión como el desarrollo de indicadores de calidad propiciarán un mejor manejo del recurso y de la agricultura sostenible, así como la toma de decisiones acertadas y el establecimiento de políticas adecuadas para su uso (Petruzelli 2000).

La calidad del suelo se interpreta como la utilidad que tiene el recurso para un propósito específico en una escala amplia de tiempo (Carter et al. 1997); como una medida de su capacidad para funcionar adecuadamente con relación a un uso específico (Gregorich et al. 1994), o como su capacidad para funcionar dentro de los límites de un ecosistema natural o manejado, sostener la productividad de la flora y la fauna, mantener o elevar la calidad del aire y del agua y preservar la salud humana y el hábitat (Karlen et al. 1994, 1997).

La salud del suelo es un aspecto que actualmente ha mermado, principalmente por la actividad agrícola intensiva, la cual incorpora de forma desmedida agroquímicos biodegradadores (principalmente plaguicidas) que provocan la modificación de las propiedades físicas, químicas y bioquímicas del suelo, lo que representa un riesgo para el mantenimiento del ecosistema y de las poblaciones humanas. Lo preocupante es que la tendencia es global, es decir, 
se trata de un problema de amplias dimensiones que merece atención regional, nacional e internacional oportuna y pertinente.

La producción agrícola en el Nevado de Toluca (NT), México (como en otras latitudes del contexto nacional e internacional) se caracteriza por el predominio de la tendencia hacia la máxima rentabilidad a corto plazo. Esto se debe, en parte, al marco de referencia actual representado por los sistemas económicos que privilegian la rentabilidad inmediata, en detrimento de la planeación a largo plazo, la cual es castigada por la alta tasa de descuento y el valor de oportunidad del dinero, que por lo general es muy alto.

Lo anterior pone de manifiesto que existe una planeación a corto plazo, porque el costo de oportunidad es tan grande que no conviene esperar. Entre más rápido se conviertan los recursos naturales en dinero, la rentabilidad será mayor. La consecuencia es que se están minando los recursos, en lugar de usarlos y conservarlos. Lo más elocuente es que deben propiciarse cambios mediante herramientas que consideren todos los costos, incluso los ambientales y transgeneracionales, y con ello garantizar la transición hacia el desarrollo sostenible (Parr et al. 2002, Porta et al. 2003). Se requiere un cambio fundamental en la manera de detonar el desarrollo; es decir, se necesita promover el desarrollo visto en su dimensión social de largo plazo y en su contexto más amplio.

En la zona del NT se practica agricultura intensiva, agricultura semiintensiva y agricultura tradicional para la producción de papa (Solanum tuberosum); sin embargo, la agricultura intensiva predomina consistentemente en la región. Dicha producción incluye la aplicación excesiva de agroquímicos (plaguicidas-fertilizantes) e incesante laboreo, lo cual está conduciendo a la degradaciónerosión consistente del suelo, ya que se han modificado significativamente sus propiedades químicas $(\mathrm{pH}$, materia orgánica y capacidad de intercambio catiónico, entre otras) y bioquímicas (Martínez-Alva et al. 2015).

El problema más relevante de los plaguicidas depositados en el NT radica en que pueden acumularse en los suelos andosólicos característicos de la zona, los cuales pueden geodisponerse con relativa facilidad debido a la carga abundante de agua en la región y la acidez característica (Corinne et al. 2006, Martínez-Alva et al. 2015). Cuando se supera la capacidad de retención de un suelo y pierde la capacidad de inmovilizar los contaminantes mediante procesos de sorción y precipitación, surgen factores de riesgo potencial para el ambiente y la salud humana (Li y Shuman 1996, Virkutyte et al. 2002, Mahler 2003, García y Dorronsoro 2005, Corinne et al. 2006, Carvalho et al. 2009).

La presente investigación está enmarcada en el contexto del desarrollo social sostenible y el conocimiento de los efectos que surgen del laboreo del cultivo de papa y las practicas intensivas y semiintensivas de producción. El objetivo del presente trabajo de investigación fue determinar la concentración total y geodisponible de elementos potencialmente tóxicos (EPT) tales como As, $\mathrm{Pb}$ y Cd (que son los elementos indicados con más frecuencia en las etiquetas de los plaguicidas empleados en la producción de papa), depositados en los suelos agrícolas de tipo Andosol Úmbricos, Regosol Eútricos y Feozem de vocación intensiva y semiintensiva para la producción de papa en la comunidad de La Peñuela, Nevado de Toluca, Zinacantepec, Estado de México, México (enero de 2019).

La zona de estudio se ha monitoreado desde 2011 con el propósito de identificar los cambios de disponibilidad y geodisponibilidad de EPT que puedan representar un riesgo inminente para la salud de los habitantes de la región e incidir tanto en las políticas productivas de alimentos como en las políticas públicas de esta región natural prioritaria para el Estado de México.

\section{MATERIALES Y MÉTODOS}

\section{Descripción de la zona de estudio}

E1 NT es un área natural protegida que abarca 46 784 ha, ubicada en el sureste del Valle de Toluca, Estado de México. Está incluido en la provincia fisiográfica denominada Sistema Neovolcánico Transversal entre las coordenadas geográficas $18^{\circ} 51^{\prime} 31^{\prime \prime}$ y $19^{\circ} 19^{\prime} 03^{\prime \prime}$ latitud norte, y $99^{\circ} 38^{\prime} 54^{\prime \prime}$ y $100^{\circ} 09^{\prime}$ 58 " longitud oeste (CONANP 2008). La zona incluye los municipios mexiquenses de Almoloya de Juárez, Amanalco de Becerra, Calimaya, Coatepec Harinas, Temascaltepec, Tenango del Valle, Texcaltitlán, Toluca, Villa Guerrero, Villa Victoria y Zinacantepec (GEM 2011).

El NT o Xinantécatl se distingue por su pico de altitud de 4680 msnm. Debido a su geomorfología y a su rango altitudinal predominante en proyección (mayor a $3000 \mathrm{msnm}$ ), descienden numerosos arroyos que contribuyen al origen de las cuencas del río Lerma-Chapala-Santiago al norte y al oriente, y del río Balsas al sur y al occidente (CONAGUA 2008). Cerca de 50 arroyos permanentes descienden del 
NT en cuatro direcciones. Estos cuerpos de agua albergan una alta biodiversidad debido a factores como la humedad, la conformación topográfica del área y el tipo de vegetación que se establece en las orillas de los cauces (CONABIO 2004).

Con base en su formación geológica (roca encajonante de caolinita-pirita) y los criterios de clasificación de la Organización de las Naciones Unidas para la Alimentación y la Agricultura (FAO) y la Organización de la Naciones Unidas para la Educación, la Ciencia y la Cultura (UNESCO), el NT se caracteriza por tener suelos de tipo Andosol Úmbricos, Regosol Eútricos y Feozem. El clima predominante es frío en la cima del volcán, semifrío con régimen de lluvias en verano en las laderas, y subhúmedo y templado con lluvias en verano en los límites inferiores. La temperatura media fluctúa entre 2 y $7{ }^{\circ} \mathrm{C}$, y registra precipitaciones entre 700 y $1000 \mathrm{~mm}$ anuales, aproximadamente (CONANP 2008). El principal cultivo del NT es la papa, que en general requiere de una amplia variedad de agroquímicos debido a múltiples factores como la proliferación de agentes causales de diversas enfermedades y la necesidad de potencializar su producción, entre otros (Porta 2003). Eventualmente se producen avena, flor de nube y calabaza (Franco et al. 2006).

La zona de estudio se caracteriza por una alta diversidad biológica micológica, florística y faunística. La vegetación predominante en el NT son los bosques de pino (Pinus pseudostrobus), pino-encino (Pinus pseudostrobus y Quercus laurina), oyamel (Abies religiosa) y pasto alpino. Entre la fauna de la zona se encuentran aproximadamente 92 especies de vertebrados. No obstante, la región se distingue por su carga específica de especies endémicas de hongos, flora y fauna (Franco et al. 2006).

La comunidad de La Peñuela (769 ha) es una de las 54 localidades del municipio de Zinacantepec, Estado de México, y una de las 23 localidades que se encuentran al interior del NT (anteriormente denominado Parque Nacional Nevado de Toluca). Se localiza a los $99^{\circ} 51^{\prime} 8.58^{\prime \prime}$ de longitud oeste y $19^{\circ}$ 9' 35.9" de latitud norte, a una altura de $3040 \mathrm{msnm}$, justo en el límite inferior del NT.

El clima (C [E] wig) es semifrío subhúmedo con temperatura media anual de 2 a $7^{\circ} \mathrm{C}$. El régimen de lluvias en verano es isotermal y la temperatura del mes más caliente se presenta antes del solsticio de verano. Este tipo de clima rodea todo el NT en la franja de los 2800 a $3700 \mathrm{~m}$ de altitud y se define en la parte norte $y / 0$ sureste de la zona de estudio (GEM 2011).

\section{Muestras y análisis de laboratorio}

Los sitios de muestreo se ubicaron con un dispositivo GPS conforme al sistema que utiliza actualmente el Instituto Nacional de Estadística y Geografía (INEGI) de México, en coordenadas UTM-WGS84. Dichos sitios se seleccionaron de acuerdo con la dirección del viento y el agua, que fluyen de norte a sur y son las vías de dispersión de los suelos en la zona.

Se muestrearon en total 40 parcelas que se usan para el cultivo de papa según los criterios propuestos por Davor (2005). Se empleó la siguiente identificación de los tipos de suelo de las parcelas muestreadas: suelos de vocación intensiva (SVI), suelos de vocación semiintensiva (SVS-I) y suelos de vocación tradicional (SVT). El control fueron suelos boscosos conservados asociados con los suelos evaluados (20 muestras compuestas). Las definiciones fueron las siguientes:

SVI: se caracterizan por el abuso del uso de agroquímicos (generalmente se incorpora más del doble de la dosis señalada en la etiqueta), por los altos rendimientos productivos, por la nula incorporación de materia orgánica $(\mathrm{MO})$, por la nula rotación del cultivo y por el alto laboreo mecanizado.

SVS-I: se caracterizan por el uso de agroquímicos (generalmente se respeta la dosis señalada en la etiqueta), por los rendimientos moderados en la producción, por el aporte ocasional de $\mathrm{MO}$, por la ocasional rotación del cultivo y por el moderado laboreo mecanizado.

SVT: se caracterizan por la nula o casi nula incorporación de agroquímicos, por los rendimientos moderados de la producción (generalmente menores a los de SVS-I), por el aporte frecuente de MO, por la rotación de cultivos y por el moderado laboreo con herramientas de tracción animal y/o manual.

En cada una de las 40 parcelas se tomaron 10 muestras simples que se mezclaron para formar una única muestra compuesta por parcela $(500 \mathrm{~g}$ de muestra). De esta manera fueron analizadas 40 muestras compuestas, 20 por cada tipo de suelo (SVI y SVS-I). Las muestras de suelo se recolectaron en enero de 2019 siguiendo los criterios que establece la Norma Mexicana NMX-AA-132-SCFI-2006 (SCFI 2006) en el horizonte Ap $(0-25 \mathrm{~cm})$, con una pala de punta hecha de aluminio (la cual se lavaba con agua corriente y se secaba con papel secante después de la toma de cada muestra para evitar la contaminación cruzada) en una distribución de tresbolillo.

Las muestras se embalaron en bolsas de plástico herméticas, se etiquetaron y se transportaron al laboratorio, donde se secaron en horno a $90^{\circ} \mathrm{C}$ durante $48 \mathrm{~h}$. Se retiraron piedras, hojas y otros materiales que no 
eran suelo. Se cuartearon y dividieron en dos partes iguales: una se resguardó como "retenido original" y el resto del suelo se utilizó para las evaluaciones. El suelo para evaluar se homogeneizó y molió con un mazo de madera cubierto de plástico y posteriormente se tamizó a través de una malla del número $10(2000 \mu \mathrm{m})$ para los análisis físicos y químicos.

En todas las muestras se determinaron las siguientes propiedades edáficas: textura, $\mathrm{pH}$ en agua en relación suelo-solución 1:2.5 y pH en $\mathrm{KCl}$ por el método propuesto por McLean (1982), conductividad eléctrica (CE) en relación sólido-agua de 1:5, porcentaje de MO por el método de Walkey y Black (1947) y capacidad de intercambio catiónico (CIC) por el método del acetato de amonio.

El análisis de la concentración total y geodisponible de los EPT de interés (As, Pb y Cd) se realizó mediante espectroscopia de absorción atómica por generación de hidruros (AA-GH) en el caso del As y mediante espectroscopia de emisión atómica por plasma de acoplamiento inductivo (ICP-AES) en el caso de los metales pesados ( $\mathrm{Pb}$ y $\mathrm{Cd}$ ). Para definir la situación de los EPT en este estudio se consideraron las concentraciones de referencia establecidas en la Norma Oficial Mexicana NOM-147-SEMARNAT/ SSA1-2007 (SEMARNAT 2007).

El procedimiento de digestión ácida se realizó de acuerdo con lo indicado en la Norma Oficial Mexicana antes citada (SEMARNAT 2007) para la extracción del elemento total. Se tomaron por cuarteo aproximadamente $5 \mathrm{~g}$ de muestra, se trituraron en un molino de bolas de óxido de zirconio y se pasaron por una malla 100 (tamaño de partícula $<149 \mu \mathrm{m}$ ). Se pesaron aproximadamente $0.5 \mathrm{~g}$ por triplicado de la muestra dentro de vasos de teflón del microondas. Se preparó un blanco y un blanco adicionado, también por triplicado. Al blanco adicionado se le añadieron $0.5 \mathrm{~mL}$ de una disolución estándar de $100 \mathrm{mg} / \mathrm{L}$ de Ag, Al, As, B, Ba, Be, Bi, Ca, Cd, Co, $\mathrm{Cr}, \mathrm{Cu}, \mathrm{Fe}, \mathrm{Ga}, \mathrm{In}, \mathrm{K}, \mathrm{Li}, \mathrm{Mg}, \mathrm{Mn}, \mathrm{Na}, \mathrm{Ni}, \mathrm{Pb}, \mathrm{Se}$, Tl, V y Zn. Posteriormente se adicionaron a cada muestra $10 \mathrm{~mL}$ de $\mathrm{HNO}_{3}$ concentrado. El sistema se dejó abierto durante una hora para permitir que las reacciones entre el ácido nítrico y los carbonatos de la muestra concluyeran (potencialmente presentes). De ese modo se evitó la formación de $\mathrm{CO}_{2}$ durante la digestión. Se cerraron los vasos y se colocaron en el carrusel. En la primera etapa de la digestión se elevó la temperatura hasta $175^{\circ} \mathrm{C}$ durante $25 \mathrm{~min}$ a una potencia de $400 \mathrm{~W}$. La segunda etapa consistió en mantener la misma temperatura durante $15 \mathrm{~min}$ adicionales. Terminada la digestión, las muestras se filtraron con papel Whatman núm. 42 y se aforaron a $100 \mathrm{~mL}$ con agua desionizada. Para la extracción del elemento geodisponible, las muestras se dejaron a saturación durante $24 \mathrm{~h}$ con agua desionizada; posteriormente se filtraron y aforaron a $25 \mathrm{~mL}$ y se tomó una alícuota de esta disolución para su análisis.

Los valores de las muestras duplicadas indicaron que la repetibilidad varía de 3 a $9 \%$. Adicionalmente, se realizó un análisis de varianza y una prueba de Tukey $(\mathrm{P}<0.05)$ de los EPT por tipo de suelo con el paquete estadístico SAS (SAS 1988), de acuerdo con el diseño experimental.

\section{RESULTADOS Y DISCUSIÓN}

\section{Propiedades físicas y químicas}

Los resultados de las propiedades físicas y químicas que se determinaron en los suelos evaluados del NT se presentan en los cuadros I y II, respectivamente, considerando dos de los tres tipos de suelos de la zona: SVI y SVS-I.

CUADRO I. TEXTURADE LOS SUELOS AGRÍCOLAS DEL NEVADO DE TOLUCA.

\begin{tabular}{lcccl}
\hline $\begin{array}{l}\text { Vocación } \\
\text { Agrícola }\end{array}$ & Arena & $\begin{array}{c}\text { Limo } \\
\%\end{array}$ & Arcilla & Tipo \\
\hline SVI & 55 & 20 & 25 & Franco arenoso \\
SVS-I & 47 & 30 & 23 & Franco arenoso \\
\hline
\end{tabular}

SVI: suelos de vocación intensiva, SVS-I: suelos de vocación semiintensiva.

Fuente: elaboración propia.

CUADRO II. PARÁMETROS QUÍMICOS DE LOS SUELOS AGRÍCOLAS DEL NEVADO DE TOLUCA.

\begin{tabular}{lccccc}
\hline $\begin{array}{l}\text { Vocación } \\
\text { agrícola }\end{array}$ & $\begin{array}{c}\mathrm{pH} \\
\mathrm{H}_{2} \mathrm{O}\end{array}$ & $\begin{array}{c}\mathrm{pH} \\
\mathrm{KCl}\end{array}$ & $\begin{array}{c}\mathrm{CE} \\
\mu \mathrm{S} / \mathrm{cm}\end{array}$ & $\begin{array}{c}\mathrm{MO} \\
\%\end{array}$ & $\begin{array}{c}\mathrm{CIC} \\
\mathrm{cmol} / \mathrm{kg}\end{array}$ \\
\hline \multicolumn{7}{c}{ medias } \\
SVI & $4.19^{\mathrm{a}}$ & $4.18^{\mathrm{a}}$ & $479^{\mathrm{a}}$ & $1.65^{\mathrm{a}}$ & $40^{\mathrm{a}}$ \\
SVS-I & $4.16^{\mathrm{a}}$ & $4.2^{\mathrm{a}}$ & $488^{\mathrm{a}}$ & $4.28^{\mathrm{b}}$ & $26^{\mathrm{b}}$ \\
\hline
\end{tabular}

CE: conductividad eléctrica, MO: materia orgánica, CIC: capacidad de intercambio catiónico, SVI: suelos de vocación intensiva, SVS-I: suelos de vocación semiintensiva. Nota: letras diferentes indican diferencias significativas. Fuente: elaboración propia.

Los suelos evaluados se definen texturalmente como francos por su contenido semiequilibrado de arena, limo y arcilla (FAO 1990). Sin embargo, pueden definirse con rigor como franco arenosos (lo 
que los hace susceptibles de encalamiento) debido a la intemperización consistente de las arcillas. Esto posibilita que la aireación y permeabilidad del recurso sean menores; asimismo, provoca la acidificación del suelo, ya que al minarse la carga específica de arcilla (así como de $\mathrm{MO}$ ), se elimina el $\mathrm{H}^{+}$a cambio de cationes como calcio $\left(\mathrm{Ca}^{2+}\right)$, magnesio $\left(\mathrm{Mg}^{2+}\right)$ y potasio $\left(\mathrm{K}^{+}\right)$, entre otros.

Los suelos evaluados son eminentemente ácidos (Cuadro II); sin embargo, ha ocurrido un cambio en los valores de $\mathrm{pH}$ en los últimos años de 3.18 (2011) en suelos agrícolas de vocación intensiva y semiintensiva (Martínez et al. 2015) a 4.17 en los mismos suelos (SVI y SVS-I) en la actualidad (enero de 2019). Es importante resaltar que los suelos evaluados tienden a un $\mathrm{pH}$ de 5.5 consistente en los suelos boscosos asociados.

La situación ácida de los suelos evaluados es una consecuencia del abuso en el uso de plaguicidas con alto contenido de EPT en la zona, ya que los sulfatos de los metales que contienen dichos plaguicidas se hidrolizan y liberan protones, lo cual afecta de modo significativo la geodisponibilidad de ciertos metales como el $\mathrm{Pb}$ y $\mathrm{Cd}$, entre otros, y la asimilación de nutrimentos. En consecuencia, se ejerce una fuerte influencia sobre la estructura del suelo y su dinámica global interactiva, principalmente con sales y metales disponibles. Se asume que dicha situación ácida influye directamente en las poblaciones de la microbiota edáfica y los hongos, los cuales determinan la disponibilidad de nutrimentos para las plantas; por ejemplo, cuando el suelo es ácido ( $\mathrm{pH}$ de 4.5 y 5.5) la descomposición de la MO hacia la producción de amoniaco (amonificación) se acelera debido a la acción de bacterias amonificantes. Por otro lado, el proceso de nitrificación es óptimo a pH de 6.5 y 7.6 (González-Fernández et al. 2003). En este sentido, se está perdiendo el equilibrio de nitrificación en la zona, dado que los suelos evaluados son eminentemente ácidos $(\mathrm{pH}=4.1)$, y su amonificación aumenta ostensiblemente.

La importancia práctica de esto radica en que se acumulan compuestos amoniacales cuando el $\mathrm{pH}$ del suelo es menor a 5.5, como es el caso. Esto es, el nitrógeno de las fuentes nítricas es convertido en amoniaco $\left(\mathrm{NH}_{3}\right)$. Cuando las condiciones del clima son adecuadas para el crecimiento del cultivo (papa) y el pH se encuentra entre 6 y 6.5, la amonificación y la nitrificación se producen casi a la misma velocidad provocando un equilibrio entre las fuentes nítrica $\left(\mathrm{NO}_{3}{ }^{-}\right)$y amoniacal $\left(\mathrm{NH}_{3}\right)$ de nitrógeno, una situación deseable en la zona, razón por la cual se adiciona cal $\left(\mathrm{CaCO}_{3}\right)$ a los suelos con el propósito de alcalinizarlos y que tiendan al equilibrio amonificación-nitrificación. Sin embargo, aún no se ha evaluado el efecto de la cal sobre el $\mathrm{pH}$ de los suelos de la zona y su recuperación potencial (no encalar los suelos con tendencia ácida puede provocar que la aplicación de plaguicidas de reacción crítica los acidifiquen aún más a través de los años).

Es importante precisar que la acidez de los suelos de la zona también puede deberse a las condiciones climáticas prevalentes (además de los plaguicidas y el origen volcánico de los suelos del NT), ya que es bien sabido que la lluvia (ácida), el viento y la temperatura controlan tanto la intensidad de lixiviación (lo cual es característico de suelos húmedos como los Andosol, Regosol y Feozem del NT) como el desgaste de los minerales del suelo. Esto influye de manera importante en las propiedades químicas del recurso, particularmente en la acidez y la salinidad (González-Fernández et al. 2003); pero, sobre todo, en la pérdida de la vegetación asociada (no conífera), que definitivamente es un factor determinante en el control de la acidez del suelo (Roberts et al. 1999).

Dicha acidez provoca la disminución del porcentaje de MO, lo que genera a su vez la disminución de la CIC del recurso (provocando generalmente la carencia de Ca y $\mathrm{Mg}$ ), la pérdida de su capacidad de adsorción y el aumento de la CE, ya que se solubilizan cationes (Cuadro II). Además, produce un incremento notable de la geodisponibilidad de elementos como $\mathrm{Al}, \mathrm{Pb}, \mathrm{y} \mathrm{Cd}$, entre otros, causando efectos adversos sobre la microbiota edáfica, hongos, plantas, animales y cualquier otro organismo que dependa del sistema.

En particular, el Al y el Cd forman un complejo de esfera interna muy estable al interactuar con el agua (presente en los horizontes superficiales) . E1 producto de su hidrólisis es un ácido moderadamente fuerte con un pKa de 3.3, lo que aumenta aún más la acidez. Normalmente, la interacción del Al con el $\mathrm{Cd}$ y agua provoca efectos directos indeseables en el metabolismo de la plantas, como interferencia en la transferencia de iones y agua a través de las membranas celulares de la raíz. Las raíces se acortan y engrosan, lo que afecta la habilidad de la planta para absorber agua y nutrimentos, particularmente fosfato.

Los valores de CE de los suelos evaluados (479 y $488 \mu \mathrm{S} / \mathrm{cm}$ para SVI y SVS-I, respectivamente) no tuvieron diferencias significativas. Son evidencia de la carga específica de sales y de la acidez de los suelos, y también son evidencia contundente de la presencia de diferentes especies químicas de metales pesados y arsénico. En el caso de la MO, la condición es consistente, dado que en ambos tipos de suelos evaluados se redujo el porcentaje total de esta 
materia, lo que representa bajos rendimientos en el cultivo y saturación de bases intercambiables en el mínimo perfil edáfico. La carencia de $\mathrm{MO}$ en los suelos evaluados evita que los oxianiones de metales pesados sean reducidos a la forma trivalente, ya que al carecer de este donador de electrones $u$ otros compuestos reductores inorgánicos asociados, la dinámica global del suelo es impactada negativamente, sobre todo a nivel de la movilidad de los micronutrimentos y la vida microbiana (Bain 2000).

\section{Concentración geodisponible de EPT}

En los cuadros III, IV y V se presenta la concentración total y geodisponible de los EPT evaluados (As, $\mathrm{Pb}$ y $\mathrm{Cd}$ ) en los SVI y SVS-I considerados, los cuales se caracterizaron en este estudio por su alta concentración total de EPT en todos los casos (As, $\mathrm{Pb}$ y Cd) sin diferencia alguna. Estos puede deberse a que en ambos tipos de suelo se abusa del uso de plaguicidas para la producción de papa (Cuadros III-V).

CUADRO III. CONCENTRACIÓNTOTALY GEODISPONIBLE DE As EN SUELOS AGRÍCOLAS DEL NEVADO DE TOLUCA.

\begin{tabular}{lcc}
\hline $\begin{array}{l}\text { Vocación } \\
\text { agrícola }\end{array}$ & $\begin{array}{c}\text { Total } \\
(\text { medias en } \\
\mathrm{mg} / \mathrm{kg})\end{array}$ & $\begin{array}{c}\text { Geodisponible } \\
(\mathrm{mg} / \mathrm{L}) \\
\text { medias }\end{array}$ \\
\hline SVI & $9.98^{\mathrm{a}}$ & 0.09 \\
SVS-I & $9.66^{\mathrm{a}}$ & 0.08 \\
\hline
\end{tabular}

BLD: bajo el límite de detección (total $=4 \mathrm{mg} / \mathrm{kg}$, soluble = $0.05 \mathrm{mg} / \mathrm{L}$ ), valor de fondo geodisponible: $\mathrm{As}=0.05 \mathrm{mg} / \mathrm{L}$, SVI: suelos de vocación intensiva, SVS-I; suelos de vocación semiintensiva.

Fuente: elaboración propia.

CUADRO IV. CONCENTRACIÓN TOTAL Y GEODISPONIBLE DE Pb EN SUELOS AGRÍCOLAS DEL NEVADO DE TOLUCA.

\begin{tabular}{lcc}
\hline $\begin{array}{l}\text { Vocación } \\
\text { agrícola }\end{array}$ & $\begin{array}{c}\text { Total } \\
(\mathrm{mg} / \mathrm{kg})\end{array}$ & $\begin{array}{c}\text { Geodisponible } \\
(\mathrm{mg} / \mathrm{L})\end{array}$ \\
\hline SVI & $398^{\mathrm{a}}$ & 0.09 \\
SVS-I & $393^{\mathrm{a}}$ & 0.09 \\
\hline
\end{tabular}

BLD: bajo el límite de detección (total $=200 \mathrm{mg} / \mathrm{kg}$, soluble $=0.05 \mathrm{mg} / \mathrm{L}$ ), valor de fondo geodisponible: $\mathrm{Pb}=0.06 \mathrm{mg} / \mathrm{L}$, SVI: suelos de vocación intensiva, SVS-I; suelos de vocación semiintensiva.

Fuente: elaboración propia.

En una investigación realizada en la zona, Martínez-Alva et al. (2015) encontraron que la concentración total de As y Cd no fueron diferentes
CUADRO V. CONCENTRACIÓN TOTAL Y GEODISPONIBLE DE Cd EN SUELOS AGRÍCOLAS DEL NEVADO DE TOLUCA.

\begin{tabular}{lcc}
\hline $\begin{array}{l}\text { Vocación } \\
\text { agrícola }\end{array}$ & $\begin{array}{c}\text { Total } \\
(\mathrm{mg} / \mathrm{kg}) \text { medias }\end{array}$ & $\begin{array}{c}\text { Geodisponible } \\
(\mathrm{mg} / \mathrm{L}) \text { medias }\end{array}$ \\
\hline SVI & $4.89^{\mathrm{a}}$ & 0.09 \\
SVS-I & $4.74^{\mathrm{a}}$ & 0.08 \\
\hline
\end{tabular}

BLD: bajo el límite de detección (total $=$ no reportado, soluble $=0.04 \mathrm{mg} / \mathrm{L}$ ), valor de fondo geodisponible: $\mathrm{Cd}=0.04 \mathrm{mg} / \mathrm{L}$, SVI: suelos de vocación intensiva, SVS-I; suelos de vocación semiintensiva.

Fuente: elaboración propia.

significativamente en los suelos de agricultura intensiva $(\mathrm{As}=9.54 \mathrm{mg} / \mathrm{kg}$ y Cd $=3.49 \mathrm{mg} / \mathrm{kg})$ y semiintensiva ( $\mathrm{As}=8.61 \mathrm{mg} / \mathrm{kg}$ y Cd$=2.11 \mathrm{mg} / \mathrm{kg})$. En ambos casos, los valores geodisponibles estuvieron por debajo del límite de detección. No obstante, en la actualidad la presencia de As y el $\mathrm{Cd}$ es notoria en la fracción geodisponible (Cuadros III y V), lo que constituye evidencia contundente de que se están depositando cada vez más EPT que representan un riesgoso potencial para la salud de la población y el ambiente. La misma situación se observa con el $\mathrm{Pb}$.

Es importante precisar que la concentración geodisponible de As y Cd que se determinó en los suelos evaluados puede producir efectos potencialmente adversos sobre la salud de la población en el corto plazo. En el caso particular del As, se sabe que concentraciones superiores a $50 \mu \mathrm{g}$ As/g de creatinina en orina pueden provocar náusea, vómito y diarrea, disminución en la producción de eritrocitos y leucocitos, ritmo cardiaco anormal, daños a los vasos sanguíneos, alteraciones en el sistema nervioso, sensación de hormigueo en manos y pies, y eventualmente la muerte (ATSDR 2007a).

La exposición crónica a As en agua para consumo humano también está relacionada positivamente con el incremento en el riesgo de padecer cáncer de riñón, vejiga, piel y pulmón (Woo y Chi 2001). En este sentido, se asume que el consumo de suelo (ordinariamente en niños) también puede estar relacionado con el riesgo de padecer dichos tipos de cáncer. La exposición a As y metales pesados $(\mathrm{Pb}$ y Cd) ha sido una constante en la zona desde 2011 (Martínez-Alva et al. 2015), cuando se empezó a evaluar la concentración total y geodisponible de EPT en los suelos del NT. Esta circunstancia justifica la pronta evaluación de la concentración total y geodisponible de As en los cuerpos de agua de la zona y sedimentos, así como la biodisponibilidad de dichos EPT en flora, fauna y población humana 
(biodisponibilidad momentánea y su correlación con daño genético y citotóxico), con el propósito de diagnosticar con alta precisión científica la situación de esta valiosa región del NT e incidir positivamente en la atención del problema.

En el caso del $\mathrm{Cd}$, la exposición crónica a este metal pesado es todavía más preocupante, ya que este agente se relaciona positivamente con la promoción de daño renal contundente (nefropatía severa) y daños permanentes al sistema nervioso central, lo que puede causar distintos tipos de discapacidad en la población (principalmente por daño al riñón), y eventualmente la muerte súbita (Carvalho et al. 2009).

En el caso del $\mathrm{Pb}$ (Cuadro IV), la concentración total en ambos tipos de suelos (398 y $393 \mathrm{mg} / \mathrm{kg}$ para SVI y SVS-I, respectivamente) ha aumentado dramáticamente respecto a su evaluación en 2011 (Martínez-Alva et al 2015), ya que está por alcanzarse el límite permisible (200-400 mg/kg) establecido en la Norma Oficial Mexicana NOM-147-SEMARNAT/ SSA1-2004 (SEMARNAT 2007), lo que representa un riesgo inminente para la salud de la población y el ecosistema. Lo preocupante es que la fracción geodisponible en ambos tipos de suelos (SVI y SVS-I $=0.09 \mathrm{mg} / \mathrm{L})$ rebasa el estándar soluble $(0.05 \mathrm{mg} / \mathrm{L})$, una evidencia clara de la posible adversidad que está por sobrevenir en la región. En adultos de la zona se han detectado lesiones en la piel presumiblemente cancerosas que, sin embargo, no se han diagnosticado clínicamente. En el caso de los niños jornaleros también se han observado lesiones en piel y refieren dolores a nivel renal, un asunto preocupante que no ha recibido la atención pertinente de las autoridades de salud pública. Se presume que la carga de $\mathrm{As} \mathrm{y} \mathrm{Cd}$ podría estar relacionada con dicha situación.

En el caso de los niños, concentraciones de $\mathrm{Pb}$ de 10 a $30 \mu \mathrm{g} / \mathrm{dL}$ en sangre pueden ocasionar disminución del coeficiente intelectual, de la velocidad de conducción nerviosa y del metabolismo de la vitamina D (ATSDR 2007b). No obstante, se ha reportado que concentraciones por debajo de $10 \mu \mathrm{g} / \mathrm{dL}$ en sangre pueden causar daño neurológico en infantes, trastornos altamente incapacitantes y eventualmente la muerte (Canfield et al. 2013).

La nueva realidad que se vive en la zona de estudio debe ser atendida con pertinencia por todos los actores involucrados del estado: comunidad científica, autoridades civiles, autoridades en materia de salud pública y educadores, entre otros. Esto justifica que en el corto plazo se diseñe, implemente y evalúe un programa de comunicación de riesgos (PCR) con el propósito de disminuir la exposición a EPT (principalmente $\mathrm{As}, \mathrm{Pb}$ y $\mathrm{Cd}$ ), la cual constituye un riesgo potencial para la salud de los pobladores y para el ambiente.

\section{CONCLUSIONES}

Los plaguicidas son un factor degradante de los suelos agrícolas del NT, ya que modifican sus propiedades físicas y químicas, principalmente en los suelos evaluados de vocación intensiva y semiintensiva para el cultivo de papa.

La acidez de los suelos evaluados es un factor determinante en la disminución de la MO y la CIC, así como en el incremento de la CE (como se observó principalmente en los SVI), y propicia un aumento ostensible de la geodisponibilidad de EPT en la región.

La concentración geodisponible de los EPT obtenidos en los suelos evaluados puede representar un riesgo ambiental y para la salud de la población en el corto plazo, y agravarse en el mediano y largo plazo si persiste la tendencia al abuso del uso de plaguicidas con alta concentración de metales pesados y As en la zona.

Es imperioso diseñar, implementar y evaluar un programa de comunicación de riesgos para reducir la exposición a EPT de la población del NT, con el propósito de incidir positivamente en la calidad de vida de las personas que trabajan y viven en esta región tan importante del Estado de México.

\section{REFERENCIAS}

ATSDR (2007a). Toxicological profile for arsenic. United States Department of Health and Human Services, Agency for Toxic Substances and Disease Registry. Atlanta, GA, USA, 559 pp.

ATSDR (2007b). Toxicological profile for lead. United States Department of Health and Human Services, Agency for Toxic Substances and Disease Registry. Atlanta, GA, USA, $582 \mathrm{pp}$.

Bain J.G., Blowes D.W., Robertson W.D. y Frind E.O. (2000). Modelling of sulfoxidation with reactive transport at a mine drainage site. J. Contam. Hydrol. 41, 2347. https://doi.org/10.1016/S0169-7722(99)00069-8

Bolognesi C. (2003). Genotoxicity of pesticides: A review of human biomonitoring studies. Mutat. Res. 543 (6), 251-272. https://doi.org/10.1016/s1383$5742(03) 00015-2$

Boul S.W. (2005). Sustainability of soil use. Annu. Rev. Ecol. Evol. Syst. 26 (1), 25-44. https://doi.org/10.1146/ annurev.es.26.110195.000325 
Bull S., Fletcher K., Boobis A.R. y Battershill J.M. (2006). Evidence for genotoxicity of pesticides in pesticide applicators: a review. Mutagenesis, 21 (2), 93-103. https://doi.org/10.1093/mutage/gel011

Canfield R.L., Henderson Ch.R., Cory-Slechta D.A., Cox Ch., Jusko T.A. y Lanphear B.P. (2013). Intellectual impairment in children with blood lead concentrations below $10 \mu \mathrm{g}$ per deciliter. N. Engl. J. Med. 348, 15171526. https://doi.org/10.1056/NEJMoa022848

Carter M.R., Gregorich E.G., Anderson D.W., Doran J.W., Jansen H.H. y Pierce F. J. (1997). Concepts of soil quality and their significance. En: Soil quality for crop production and ecosystem health, vol. 3 (Gregorich E.G.. y Carter M., Eds.). Elsevier Science Publishers, Amsterdam, Holanda, 213-258.

Carvalho A.S.C., Santos A.S., Pereira S.F.P. y Alves C.N. (2009). Levels of $\mathrm{As}, \mathrm{Cd}, \mathrm{Pb}$ and $\mathrm{Hg}$ found in the hair from people living in Altamira, Pará, Brazil: Environmental implications in the Belo Monte Area. J. Braz. Chem. Soc. 20 (6), 1153-1163. https://doi.org/10.1590/ S0103-50532009000600022

Cavallo D., Cinzia L.U., Carelli G., Iavicoli I., Ciervo A., Perniconi B., Rondinone B., Gismondi M. e IavicoLi S. (2006). Occupational exposure in airport personnel characterization and evaluation of genotoxic and oxidative effects. Toxicology 223 (5), 26-35. https:// doi.org/10.1016/j.tox.2006.03.003

CONABIO (2004). Regiones terrestres prioritarias de México. Comisión Nacional para el Conocimiento de la Biodiversidad, México [en línea]. http://www. conabio. gob.mx/informacion/metadata/gis/rtp1mgw. xml?_httpcache=yes\&_xsl=/db/metadata/xsl/fgdc html. xsl\&_indent $=$ no 06/05/2019

CONAGUA (2008). Estadísticas del agua en México. Comisión Nacional del Agua, México [en línea]. http:// www.conagua.gob.mx/CONAGUA07/Publicaciones/ Publicaciones/EAM_2008.pdf 06/05/2019

CONANP (2008). Parques nacionales. Comisión Nacional de Áreas Naturales Protegidas, México [en línea]. http://nevadodetoluca.conanp.gob.mx 06/05/2019

Corinne P.R., Fang-Jie Z. y Steve McGrath P. (2006). Phytotoxicity of nickel in a range of European soils: Influence of soil properties, Ni solubility and speciation. Environ. Pollut. 145 (2), 596-605. https://doi. org/10.1016/j.envpol.2006.04.008

Davor R. (2005). Heavy metals distribution in agricultural topsoils in urban area. Environ. Geol. 43, 795-805. https://doi.org/10.1007/s00254-002-0694-9

FAO (1990). Soil map of the world (revised legend). Food and Agriculture Organization of the United Nations, Roma, Italia. 23 pp.

Franco S., Regil H. y Ordóñez J. (2006). Dinámica de perturbación de las zonas forestales en el Parque
Nacional Nevado de Toluca. Madera y Bosque 12 (2), 17-28.

García I. y Dorronsoro C. (2005). Contaminación por metales pesados. En: Tecnología de suelos, vol. 2 (Bolonegsi G.E., Ed.). Universidad de Granada, Departamento de Edafología y Química Agrícola. Madrid, España, 145-148.

Garry V.F. (2004). Pesticides and children. Toxicol. Appl. Pharmacol. 198 (2), 152-163. https://doi.org/10.1016/j. taap.2003.11.027

GEM (2011). Áreas Naturales Protegidas en el Estado de México. Categorías de las áreas protegidas, Gobierno del Estado de México, Secretaría de Medio Ambiente, Comisión Estatal de Parques Naturales y de la Fauna.

González-Fernández P., Ordónez-Fernández R., EspejoSerrano R. y Peregrini-Alonso R. (2003). Cambios en el $\mathrm{pH}$ del perfil de un suelo ácido cultivado y enmendado con diversos materiales para incrementar su fertilidad. En: Estudios de la zona no saturada del suelo, vol. 6 (Álvarez-Benedi J. y Marinero P., Eds.). Universidad Politécnica de Madrid, Madrid, España, 373-388.

Gregorich E.G., Carter M.R., Angers D.A., Monreal C.M. y Ellert B.H. (1994). Towards a minimum data set to asses soil organic matter quality in agricultural soils. Can. J. Soil Sci. 74 (2), 367-386. https://doi. org/10.4141/cjss94-051

Haro G.L., Chaín C.T., Barrón A.R. y Bohórquez L.A. (2002). Efectos de plaguicidas agroquímicos: Perfil epidemiológico-ocupacional de trabajadores expuestos. Revista Médica del IMSS 40 (2), 19-24.

Hernández A., Hernández P. y Gordillo A. (2006). Manual para la evaluación de impactos ambientales. INNCIVE, Madrid, 770 pp.

Ismail I.B.S., Farihah K. y Khairiah J. (2008). Bioaccumulation of heavy metals in vegetables from selected agricultural areas. Bull. Environ. Contam. Toxicol. 74 (2), 320-327. https://doi.org/10.1007/ s00128-004-0587-6

Karlen D,L., Wollenhaupt N.C., Erbach D.C., Berry E.C., Swan J.B., Each N.S. y Jordahl J.L. (1994). Crop residues effects on soil quality following 10-years of no-till corn. Soil Tillage Res. 31 (2), 149-167. https:// doi.org/10.1016/0167-1987(94)90077-9

Karlen D.L., Mausbach M.J., Doran J.W., Cline R.G., Harris R.F. y Schuman G.E. (1997). Soil quality: A concept, definition and framework for evaluation. Soil Sci. Soc. Am. J. 61 (2), 4-10. https://doi.org/10.2136/ sssaj1997.03615995006100010001x

Li Z. y Shuman L.M. (1996). Heavy metal movement in metal contaminated soil profiles. Soil Sci. 161 (2), 656-666. 
Mahler R.L. (2003). General overview of nutrition for field and container crops. En: National proceeding: Forest and Conservation Nursery Associations (Riley L.E., Dumroese R.K. y Landis T.D., Eds.). Proc. RMRSP-33. U.S. Department of Agriculture, Forest Service, Rocky Mountain Research Station, 33-39.

Mansour S. (2004). Pesticide exposure-Egyptian scene. Toxicology 198 (2), 91-115. https://doi.org/10.1016/j. tox.2004.01.036

Martínez-Alva G., Gutiérrez-Ruiz M.E., Martínez-Campos A.R., Villalobos-Pietrini R. y Arteaga-Reyes T.T. (2015). Concentración total y geodisponible de elementos potencialmente tóxicos en suelos volcánicos con uso agrícola del Nevado de Toluca, México. Rev. Int. Contam. Amb. 31 (2), 113-125.

McLean E.O. (1982). Soil pH and lime requirements. En: Methods of soil analysis. Part 2. (Page A.L., Miller R.H. y Keeney D.R., Eds.). Chemical and Microbiological Properties, American Society of Agronomy, Soil Science Society of America, Madison, 199-224.

Parr J.F., Papendick R.L., Hornick S.B. y Meyer R.E. (2002). Soil quality: Attributes and relationships to alternative and sustainable agriculture. Am. J. Alternative Agr. 7 (2), 5-11. https://doi.org/10.1017/ S0889189300004367

Petruzelli G. (2000). Recycling wastes in agriculture: Heavy metal bioavailability. Agric. Ecosyst. Environ. 27 (2), 493-503. https://doi.org/10.1016/01678809(89)90110-2

Porta J., López A.M. y Roquero L.C. (2003). Edafología para la agricultura y el medio ambiente. Mundi Prensa, Madrid, 562 pp.
Roberts T.M., Skeffington R.A. y Blank, L.W. (1999). Causes of Type 1 Spruce decline in Europe. Forestry 62, 179-222. https://doi.org/10.1093/forestry/62.3.179-a SAS (1988). SAS user's guide: Statistics. Release 6.03. SAS Institute. Cary, Nueva York, EUA, 1028 pp.

SEMARNAT (2007). Norma Oficial Mexicana NOM147-SEMARNAT/SSA1-2004. Que establece los criterios para determinar las concentraciones de remediación de suelos contaminados por arsénico, bario, berilio, cadmio, cromo(vi), mercurio, níquel, plata, plomo, selenio, talio y/o vanadio y otros. Diario Oficial de la Federación, México, 2 de marzo.

USEPA (2010). Types of pesticides. United States Environmental Protection Agency [en línea]. http://www. epa.gov/pesticides/about/types.htm 06/05/2019

Virkutyte J., Sillanpää M. y Latostenmaa P. (2002). Electrokinetic soil remediation-Critical overview. Sci. Total Environ. 289 (2), 97-121. https://doi.org/10.1016/ S0048-9697(01)01027-0

Waliszewski S.M., Gómez-Arroyo S., Infanzón R.M., Carvajal O., Villalobos-Pietrini R., Trujillo P. y Maxwell Hart M. (2004). Persistent organochlorine pesticide levels in bovine fat from Mexico. Food Addit. Contam. 21 (2), 774-780. https://doi.org/10.1080/0265203041 0001712736

Walkey A.L. y Black A. (1947). A rapid determination of soil organic matter. J. Agric. Sci. 25 (2), 563-568.

Woo N.C. y Chi M.J. (2001). Arsenic and metal contamination of water resources from mining wastes in Korea. Environ. Geol. 40, 305-311. https://doi.org/10.1007/ s002540000161 